\title{
The AARL Gold Fingerprinting Technology
}

\author{
B Grigorova, S Anderson, J de Bruyn, W Smith, K Stülpner and A Barzev \\ Anglo American Research Laboratories (Pty) Ltd, ${ }^{*}$ P.O. Box 106, Crown Mines, 2025, South Africa
}

\begin{abstract}
Gold fingerprinting, as its name suggests, is a technique which provides gold profiles unique to a given source. The procedure adopts a qualitative approach based on the singular patterns produced by the minor and trace element impurities present in gold. The generation of a Gold Bullion Databank, GBD, has provided an effective means of ascertaining gold provenance in cases where the origin is unknown. The AARL fingerprinting technique is described, in particular the role of Laser Ablation Inductively Coupled Plasma Mass Spectrometry (LA-ICP-MS) in profile generation, together with the use of the AARL unique statistical model specifically designed to provide $\%$ similarity information between an unknown and members of the GBD.
\end{abstract}

The AARL gold fingerprinting technology was established in 1993 to provide a reliable means of ascertaining the provenance of gold (1). This technique, utilizing laser ablation inductively coupled plasma mass spectrometry in conjunction with an inhouse developed data processing statistical model, was designed to generate profiles corresponding to trace element distributions in gold bullion. What makes provenance studies a possibility is the fact that these profiles are unique for each individual source. The generation of gold fingerprint profiles for South African gold mines has led to the compilation of the South African Gold Bullion Databank (GBD). The fifty eight different gold sources currently contained in the GBD cover approximately $90 \%$ of the South African gold producing industry and from other African countries including Ghana, Mozambique, Mali, Zimbabwe, Tanzania and Zambia. The tentative profiles from five sources from South America are also included in the GBD.

The AARL fingerprinting technology has numerous other applications including the fingerprinting of alluvial gold grains (2), copper, precious stones, platinum group metals, powdered materials (3), ivory, rhino horn and ancient gold (4), base metals and glass artefacts.

\section{LASER ABLATION INDUCTIVELY COUPLED PLASMA MASS SPECTROMETRY (LA-ICP-MS)}

LA-ICP-MS provides an effective means of analysing solid samples with sufficient sensitivity to generate signal intensities for most elements in the Periodic Table from lithium to uranium down to sub-ppb levels.

The laser systems available at the AARL include an IR Nd:YAG laser operating at $1064 \mathrm{~nm}$ and an UV frequency quadrupled Nd:YAG laser operating at $266 \mathrm{~nm}$. The advantages of using a laser ablation system include a significant reduction of sample preparation times, opportunities for sample contamination and spectral interferences. A remote Charge Coupled Device (CCD) colour camera allows both in-situ viewing of the sample and monitoring of the actual ablation event. Crater sizes can vary from $200 \mu \mathrm{m}$, if the ablation is carried out at low magnification (50x) using the IR laser, to less than $10 \mu \mathrm{m}$, if carried out using the high magnification lens (1500x) associated with the UV system. The former would be applicable to techniques requiring bulk or representative sampling of materials while the latter would be relevant to microanalysis procedures appropriate for analysing small grains, inclusions and items such as the gold artefacts where the use of a virtually non-destructive technique is of considerable importance. 


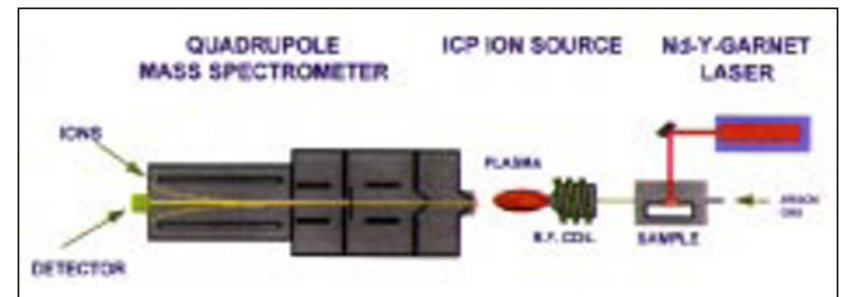

Figure 1 The laser ablation inductively coupled plasma spectrometer

A schematic diagram of the LA-ICP-MS system is provided in Figure 1. The samples are placed in a sealed quartz cell and, following ablation of the surface with the laser beam, small particles are vaporized and transported via a stream of argon to the inductively coupled plasma. The plasma is generated when free electrons, 'seeded' by a Tesla coil into the argon flow, interact with a radiofrequency magnetic field resulting in the formation of eddy currents. Resistance to the electron flow results in Joule and Ohmic heating of the gas to temperatures of approximately $7000 \mathrm{~K}$ which is sufficient to vaporize, atomize and ionize the particles passing into this region. The ions, extracted from the plasma by the sample and skimmer cones, are focused into the high vacuum quadruple mass spectrometer where they are separated on the basis of their differing mass to charge ratios. On emerging from the quadruple they strike the ion detector and a mass spectrum of signal intensity versus atomic mass is generated.

One of the major advantages of ICP-MS is the high sensitivity which can be obtained across the Periodic Table from the light elements, $\mathrm{Li}$ and $\mathrm{B}$, through to the heavier transition elements, rare earths, thorium and uranium. The wide dynamic range allows a full mass scan to be carried out for elements occurring in a concentration range from per cent right down to sub-ppb levels.

\section{THE AARL FINGERPRINTING PROCESS}

The AARL Gold Fingerprinting procedure scans 131 isotopes from ${ }^{45} \mathrm{Sc}$ to ${ }^{238} \mathrm{U}$ and covers a sufficiently large element suite to ensure that a unique fingerprint can be obtained for each individual source (Figure 2).

One of the fundamental aspects of fingerprinting is that data assessment is purely qualitative in nature due to the fact that trace element concentrations may vary significantly with refining methods and the extent of gold purity. The technique is thus based on

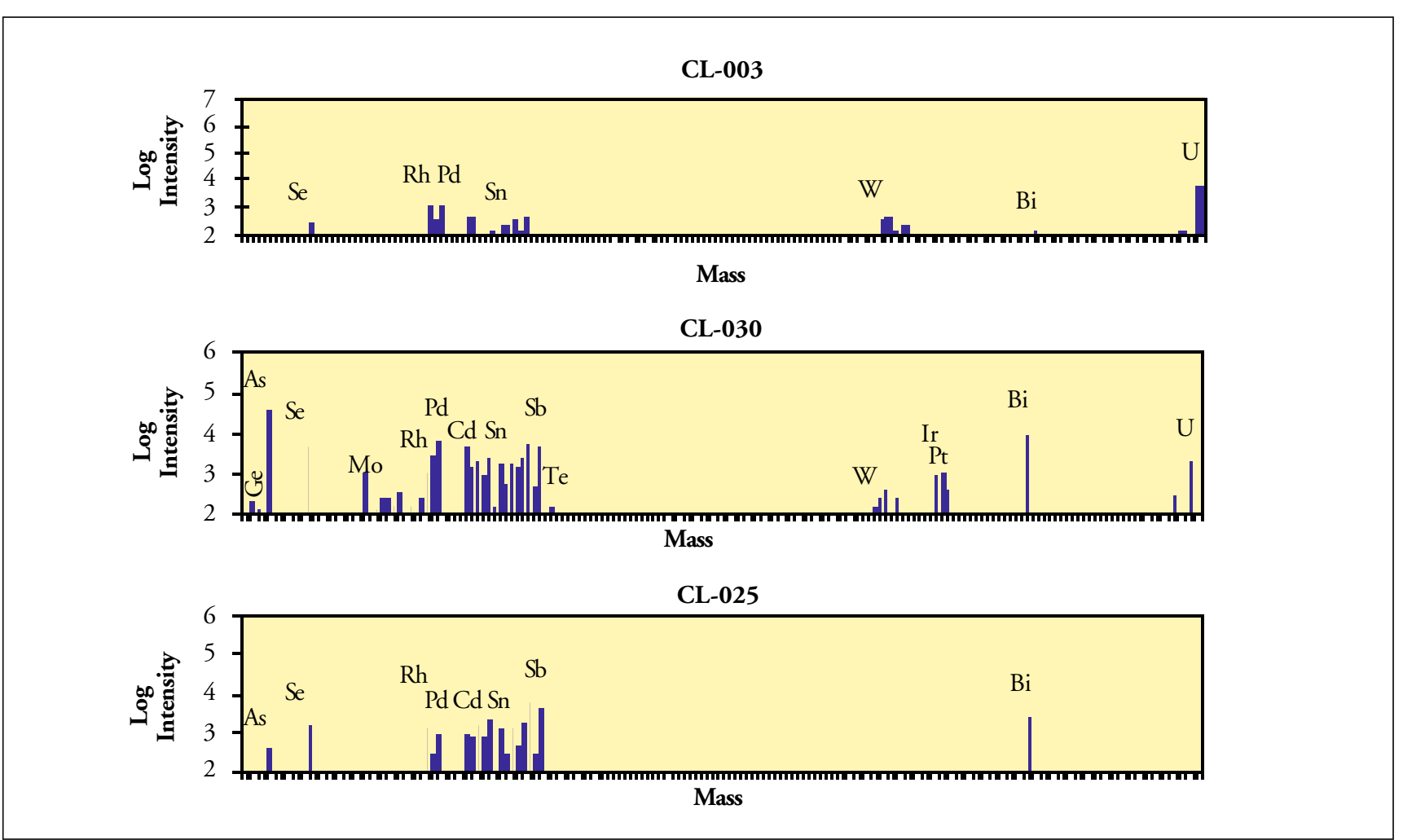

Figure 2 Mass spectral fingerprints for three adjacent gold mines. 
presence/absence patterns generated in the mass spectrum for a given source. A second means of distinguishing one source from another lies in the analysis of isotope ratios, in particular the ${ }^{206} \mathrm{~Pb},{ }^{207} \mathrm{~Pb}$ and ${ }^{208} \mathrm{~Pb}$ isotopes. The natural lead isotope ratios can vary through the radioactive decay of uranium and thorium which alters the ratios due to enrichment of $206 \mathrm{~Pb},{ }^{207} \mathrm{~Pb}$ and ${ }^{208} \mathrm{~Pb}$ (Figure 3).

The fingerprinting process is carried out in two phases. The first involves the acquisition of raw data through bulk analysis of the samples while the second entails complex data processing using the AARL unique statistical model. Prior to submission to the

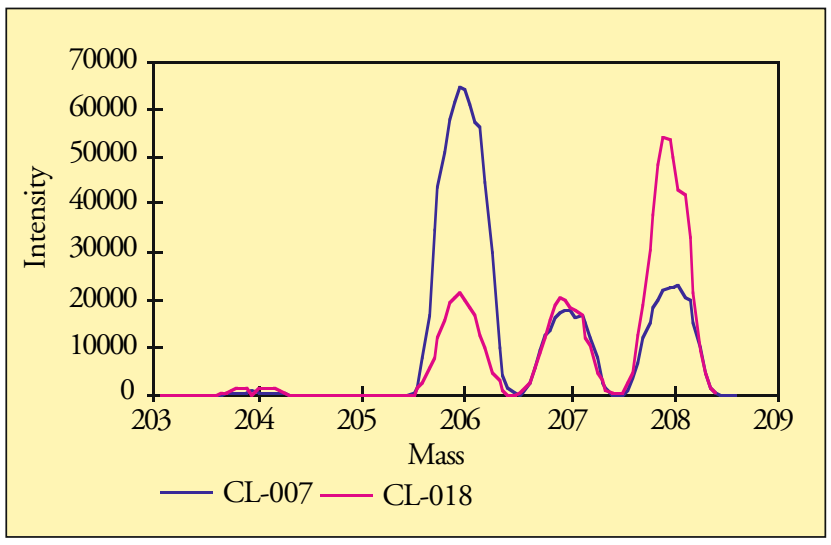

Figure 3 Lead isotope profiles of gold from two different mines. statistical model, the raw data is assessed and several procedures relating to isotope ratio calculations, blank subtraction, sensitivity correction, interference correction, secondary isotope correction, outlier rejection and normalization are carried out using a set of in-house routines. The result is a representative profile corresponding to the unknown.

The statistical model was custom built to provide a rapid and efficient means of comparing the profile of the unknown to the fifty eight source prototypes stored in the GBD and generating probability and percentage similarity information applicable to the provenance studies. The source discrimination process takes into consideration isotope ratio relationships and profile differences occurring in the trace element region of the mass spectrum. Results of the statistical model are verified by a visual comparison between the unknown and the source profiles selected. The accuracy of the identification process is illustrated in Figure 4 which contains the matched profiles of a recovered gold bullion sample and a GBD member.

\section{APPLICATIONS}

The AARL gold fingerprinting technique was initially developed to provide a valid means of ascertaining the provenance of gold recovered by the police and mine

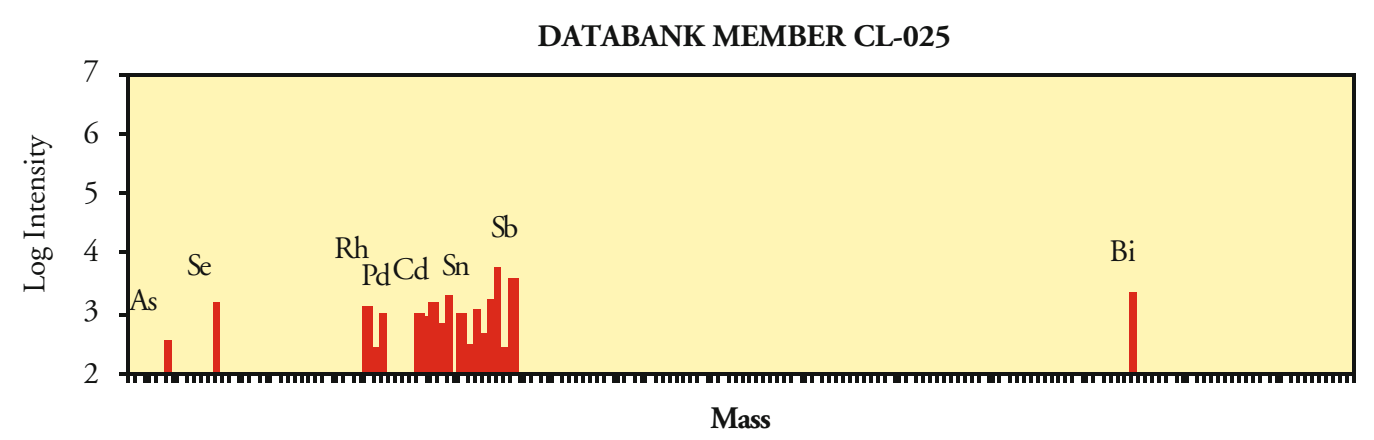

GOLD RECOVERED BY SAPS

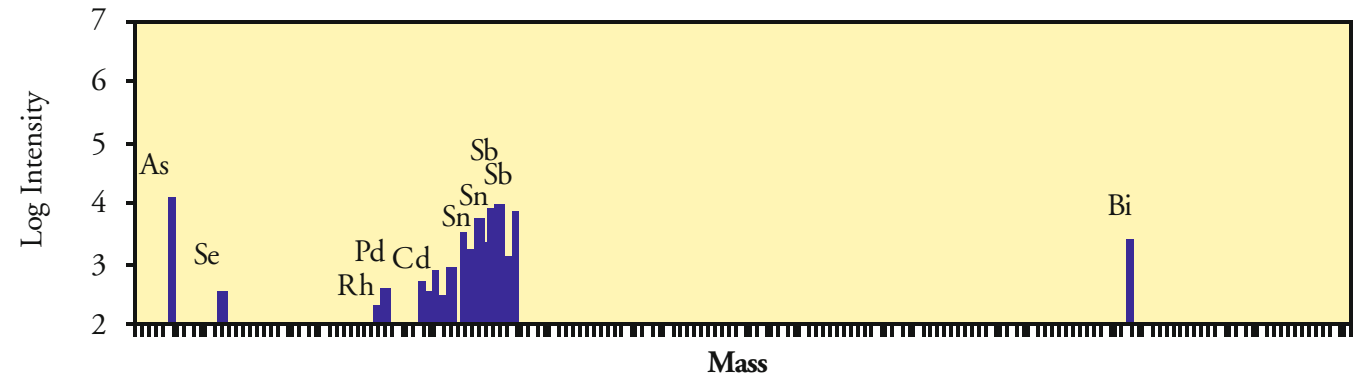

Figure 4 Comparative match between profiles of a recovered gold sample and a GBD member 
security officials, thus ensuring its return to the rightful owner, and curbing theft activities.

Other applications of the technique include the generation of fingerprint profiles for alluvial gold grains which provide geologists with information important for gold exploration activities, and the fingerprinting of platinum group metals also for provenance studies.

Recently, the fingerprinting of gold and tin artefacts from Southern African archaeological excavation sites is being used to aid in the determination of their origin, to gain more knowledge of early mining practices in this region and to identify the trade routes which existed between the various major centres. Some examples of this type of usage will be given in a later article to be published in Gold Bulletin (5).

In fact, the AARL technology has been diversified over a wide range of materials, and therefore has farreaching implications in a variety of fields, for example in the fingerprinting of other precious metals (platinum, palladium, rhodium, iridium), ivory, rhino horn, copper and crude oils, to name but a few.

\section{ABOUT THE AUTHORS}

Dara Grigorova is the Manager Research of the AARL. Dr Stan Anderson, Jan de Bruyn, Wyatt Smith and Katherine Stülpner are all members of the LA-ICP-MS research team which is headed by Alex Barzev.

\section{REFERENCES}

1 A.I. Barzev, A.E. Leins and J. de Bruyn, 'Gold Fingerprinting by Laser Ablation Inductively Coupled Plasma-Mass Spectrometry', AARL Project No. R/93/143, Report No. 1, October 1993

2 A.I. Barzev and W. Smith, 'LA-ICP-MS Analysis of Gold Grains and Data Interpretation', AARL Project No. R/94/208, Report No. 1, June 1995

3 A.I. Barzev, P.A. Winterburn and W. Smith, 'Analysis of Pathfinder Elements in Soils by LAICP-MS', AARL Project No. R/94/163, Report No. 1, February 1995

4 W. Smith, 'Report on the Fingerprinting of Gold Artefacts from Bosutswe, Mapungubwe and Thulamela Archaeological Sites', AARL Report, March 1997

5 B. Grigorova, W. Smith, K. Stülpner, J.A. Tumilty and D. Miller, Gold Bull., to be published

\section{A L E N D A R}

\section{IPMI Conferences}

The 22nd IPMI Conference will take place on June 14 - 171998 in Toronto, Ontario, Canada. The Technical Programme consists of sessions on 'Separation and Analysis', including a paper on XRF determination of gold in jewellery alloys and solvent extraction of gold cyanide; 'Finance and Economics of Precious Metals'; 'Gold', including a number of papers on gold supply, demand and production; 'Environmental Health and Safety'; 'Catalysis and Technology', including a paper on new advances in gold catalysis; and an 'Environmental Interactive Forum'.
The 23rd IPMI Conference will be held on June 20 - 231999 in Acapulco, Mexico. For further information contact
International Precious Metals Institute, 4905 West Tilghman Street, Suite 160, Allentown, Pennsylvania 181094, USA. $\mathrm{Tel}+16103959700$ $\mathrm{Fax}+16103955855$

\section{4th Eurométaux Conference}

The fourth European Precious Metals Conference, sponsored by the European Precious Metals Industry, will take place in Barcelona on the 29 - 31 March 2000.

For further information contact Monique Jones, Eurométaux, Brussels

$\mathrm{Tel}+3227756330$

Fax +3227790523 